\title{
IMPORTÂNCIA DE MICROHABITATS NA OCORRÊNCIA DE GÊNEROS DE EPHEMEROPTERA E TRICHOPTERA EM UMA ILHA NO RIO XINGU
}

\author{
Samantha Ribeiro da Silva ${ }^{*}$, Ana Luiza-Andrade ${ }^{2}$, Suyana Karla Ferreira da Silva ${ }^{1}$, Tamyris \\ Pegado de Souza e Silva ${ }^{1}$, Leandro Melo de Sousa ${ }^{1}$, Thiago Bernardi Vieira ${ }^{1}$, Leandro Schlemmer \\ Brasil $^{1}$ \& Karina Dias-Silva ${ }^{1}$
}

\begin{abstract}
${ }^{1}$ Universidade Federal do Pará, Programa de Pós-graduação em Biodiversidade e Conservação, Rua Augusto Corrêa, nº 01, Guamá, CEP 66075-110, Belém, PA, Brasil.

${ }^{2}$ Universidade Federal do Pará, Instituto de Ciências Biológicas, Programa de Pós-graduação em Zoologia, Rua Augusto Corrêa, no 01, Guamá, CEP 66075-110, Belém, PA, Brasil.

E-mails: samantharsilva20@gmail.com (*autora correspondente); andradeanaluiz@gmail.com; suyannaferreira1@ gmail.com; t.pegado@hotmail.com; leandro.m.sousa@gmail.com; thiagobernardi007@gmail.com; leandrobrasilecologia@gmail.com; diassilvakarina@gmail.com
\end{abstract}

Resumo: Este trabalho descreveu a abundância, riqueza e composição de gêneros e os grupos funcionais alimentares (GFA) de Ephemeroptera e Trichoptera (ET), em diferentes microhabitats de uma ilha fluvial, na Volta Grande do rio Xingu, Amazônia brasileira. Foram coletados insetos aquáticos em 30 amostras localizadas em poças situadas no interior da ilha (substrato de rochas); e outras 30 amostras situadas na região litorânea da ilha (substrato de seixos). As diferentes facetas da diversidade (abundância, riqueza e composição de gêneros e GFA) foram comparadas entre as amostras de poça com substrato rocha e as da região litorânea com substrato seixo. No total, foram coletados 56 indivíduos distribuídos em 15 gêneros de ET. A maior riqueza estimada de gêneros e o maior número de indivíduos ocorreram nas rochas dos ambientes de poça. A composição de gêneros diferiu entre as rochas dos ambientes de poças, e os seixos dos ambientes litorâneos, sendo a comunidade desse último mais homogênea. Existiam mais organismos raspadores distribuídos predominante nas rochas dos ambientes de poças e organismos filtradores dispostos predominantemente nos seixos dos ambientes litorâneos. A distribuição dos raspadores relacionou-se com a disponibilidade de alimento, uma vez que a ilha possui pouca vegetação e a luz sobre as rochas é propícia para a colonização por biofilme, seu principal recurso alimentar. Na região litorânea, a maior distribuição de filtradores deve-se a maior quantidade de partículas finas suspensas na água, seu principal recurso alimentar. Nossos resultados demonstraram que mesmo em ambientes espacialmente próximos, como em uma pequena ilha, as diferenças de disponibilidade de recursos alimentares em diferentes microhabitats podem influenciar na distribuição em múltiplas facetas da diversidade dos organismos estudados. Portanto, mesmo em ambientes dinâmicos como os grandes rios, a manutenção dos microhabitats é importante para as comunidades de insetos aquáticos.

Palavras-chave: Amazônia; barragens; insetos aquáticos; rocha; seixos.

IMPORTANCE OF MICROHABITATS IN THE OCCURRENCE OF EPHEMEROPTERA AND TRICHOPTERA GENERA IN AN ISLAND IN RIO XINGU. This work described the abundance, richness and composition of genera and the functional food groups (GFA) of Ephemeroptera and Trichoptera (ET), in different microhabitats of a fluvial island, in Volta Grande of the Xingu River, Brazilian Amazon. Aquatic insects were collected from 30 samples located in puddles situated in the interior of the island (rocks as 
substrate); and another 30 samples located in the coastal margin of the island (pebble as substrate). The different facets of diversity (abundance, richness and composition of genera and GFA) were compared between puddle samples with rock substrate and those from the coastal region with pebble substrate. In total, 56 individuals were collected, distributed in 15 genera ET. The greatest estimated genera richness and the largest number of individuals occurred in the rocks of the puddle environments. The composition of genera differed between the rocks in the puddle environments and the pebbles in the coastal environments, with the community of the latter being more homogeneous. There were more scraper organisms distributed predominantly in the rocks of the puddle environments and filtering organisms arranged predominantly in the pebbles of the coastal environments. The distribution of scrapers is related to the availability of food, since the island has little vegetation and the light on the rocks is favorable for colonization by biofilm, its main food resource. In the coastal region, the greater distribution of filters is related to the greater number of fine particles suspended in water, its main food resource. The results demonstrate that even in spatially close environments, such as on a small island, differences in the availability of food resources in different microhabitats can influence the distribution in multiple facets of diversity. Thus, even in dynamic environments such as large rivers, maintaining microhabitats is important for aquatic insect communities.

Keywords: Amazon; aquatic insects; dams; rock; puddles.

\section{INTRODUÇÃO}

A distribuição dos microhabitats dentro de um ecossistema aquático propicia o estabelecimento de diferentes organismos em um mesmo local. Manchas com diferentes aspectos ecológicos inseridas na paisagem, com tipos de substrato, luminosidade ou vazão distintas podem ser ocupadas por diversos grupos dentro de um corpo d`água (Thorp et al. 2006). Em ecossistemas aquáticos, nas regiões litorâneas, o rio apresenta correnteza forte, já no interior das ilhas, ocorre o predomínio de poças d'água, corpos d’água lênticos. Os organismos presentes nesses ecossistemas mais adaptados à correnteza tendem a estar mais presentes na região litorânea, enquanto que os grupos mais adaptados a ambientes lênticos nas regiões interiores de ilhas, em poças, principalmente por que existem relações entre as características intrínsecas das espécies e as condições ambientais as quais elas são expostas (Southwood 1977).

As adaptações das espécies aos hábitats estão relacionadas a restrições fisiológicas como respiração, suportar altas temperaturas e baixas concentrações de oxigenação na água, aspectos tróficos como aquisição de alimentos, ou características físicas como velocidade da água e interações bióticas como predação e competição, esses fatores são responsáveis por moldar a composição dos organismos em ambientes lênticos e lóticos (Merritt \& Cummins 1996, Juen et al. 2017). Em ambientes lóticos, a velocidade $\mathrm{e}$ o movimento das águas influenciam diretamente na disponibilidade de substratos e oxigenação da água, neste caso é esperado que os insetos coletores explorem mais este ambiente pois obtém seu alimento sem grandes gastos de energia. Outra condição que permite que alguns insetos vivam nas correntezas são as adaptações corporais dos mesmos, tais como achatamento do corpo que é típico em insetos reofílicos, e em ambientes lênticos são esperados organismos raspadores, e que sejam bons nadadores (Wallace \& Anderson 1995).

Em ambientes litorâneos com partículas de substrato pequenas a disponibilidade de microhabitats é menor e há baixa estabilidade do substrato, não havendo grande diversidade de espécies (Minshall \& Minshall 1977). Nesses locais com forte correnteza, geralmente ocorre o substrato seixo e existe maior disponibilidade de partículas finas suspensas, estes últimos são recursos alimentares para insetos aquáticos coletores filtradores (Brasil et al. 2014). Enquanto em locais onde as rochas ficam expostas à luz solar, existe maior disponibilidade de biofilme, o qual este pode ser composto por perifíton, algas e outros detritos associados, incluindo elementos microflorais e microfaunísticos, os quais são recursos alimentares para os insetos aquáticos raspadores (Cummins 1979). Além disso, os 
microhabitats com substrato de rocha permitem um ambiente mais complexo e comunidade mais diversa (Allan 1995). Portanto, microhabitats com condições e recursos diferentes são importantes preditores para a distribuição dos táxons e grupos funcionais alimentares dessas comunidades aquáticas (Shimano et al. 2012, Brasil et al. 2014).

Entre os organismos aquáticos, as ordens de insetos Ephemeroptera, Plecoptera e Trichoptera (EPT) destacam-se por desempenhar importantes papéis nas teias tróficas, ao fragmentar, coletar ou filtrar partículas orgânicas (Cummins \& Klug 1979). Além disso, são comumente utilizados em estudos de biomonitoramento, devido à alta sensibilidade às variações ambientais (Rosenberg \& Resh 1993). No entanto, a maioria dos trabalhos sobre esses grupos concentra-se nas regiões Sul e Sudeste do Brasil (Resh \& Unzicker 1975, De Marco \& Vianna 2005), a maior parte restritos à taxonomia (Shimano et al. 2013). Além disso, a maior parte dos trabalhos com EPT foram realizados em riachos de cabeceira, que tem entre um e três metros de largura, aproximadamente, desse modo, a fauna de rios de médio e grande porte, acima de $4^{\text {a }}$ ordem, e os padrões de estruturação de EPT associados a ilhas fluviais ainda são pouco conhecidos (Angeli et al. 2015). A falta de conhecimento sobre a distribuição das espécies, chamada de déficit Wallaceano, dificulta a tomada de decisão sobre conservação (Whittaker et al. 2005, Hickisch et al. 2019). Essa lacuna de conhecimento biogeográfico é maior em ambientes tropicais onde o investimento em pesquisa ainda é pequeno, diante da grande biodiversidade e extensão territorial (Barlow et al. 2018). No Brasil, essas lacunas de conhecimento são especialmente grandes se tratando de espécies aquáticas de água doce (Shimano et al. 2013, Miguel et al. 2017).

Adicionalmente à falta de conhecimento sobre a distribuição das espécies, drásticas modificações vêm ocorrendo nos sistemas fluviais, inclusive nos rios amazônicos, devido ao despejo de rejeitos de mineração, ou a construção de hidroelétricas. Um exemplo disso é a modificação da vazão hidrológica na Volta Grande do Xingu, causada pela construção da Usina Hidrelétrica de Belo Monte (Lees et al. 2016). Após a implementação da UHE Belo Monte, até $80 \%$ da vazão natural do rio pode ser desviada para o reservatório intermediário da usina e geração de energia, deixando o curso original da Volta Grande como um trecho de vazão reduzida (Norte Energia 2011). Existem previsões de que esta redução artificial cause impactos severos na biota aquática, pois altera o pulso de inundação e consequentemente vários ciclos de vida de diversos grupos animais e vegetais (Magalhães \& Hernándes 2009). Desta forma, é de fundamental importância conhecermos a estrutura das comunidades desses ambientes para que se possa averiguar possíveis alterações advindas da mudança hidrológica como consequência da operação da UHE Belo Monte.

Diante disto, evidencia-se a importância de aumentar o conhecimento sobre distribuição destas espécies, bem como compreender a influência de microhabitats nessas distribuições e, portanto, o objetivo deste trabalho foi descrever a abundância, riqueza e composição de gêneros e os grupos funcionais alimentares (GFA) de insetos aquáticos, Ephemeroptera e Trichoptera (ET), entre microhabitats litorâneos (lóticos) e de interior da ilha (lênticos), com distintos microhabitats, de uma ilha fluvial localizada na Volta Grande do rio Xingu, na Amazônia brasileira, sob influência direta do complexo Hidrelétrico de Belo Monte. Predizemos que as amostras de interior da ilha (lênticas) com substratos rochosos apresentam (1) maior riqueza de gêneros e (2) abundância de indivíduos por apresentar maior estabilidade do que amostras localizadas na região litorânea da ilha (lóticas) com substrato de seixo exposto a correnteza do rio. Além disso, esperamos que a (3) composição de gêneros também mude, uma vez que a disponibilidade distinta de recursos alimentares nos habitats filtra gêneros diferentes.

\section{MATERIAL E MÉTODOS}

\section{Área de estudo}

O estudo foi desenvolvido em uma ilha do trecho de vazão reduzida do rio Xingu, com hierarquização de drenagem acima de 5 a ordem, de acordo com o método proposto por Strahler (1957) $\quad\left(3^{\circ} 26^{\prime} 29,35^{\prime \prime}\right.$ S 51⒌'35,34" W), localizada a jusante da barragem de Pimental, no município de Vitória do Xingu (Figura 1). A ilha apesenta uma área de aproximadamente $2 \mathrm{~km}^{2}$. O rio Xingu é considerado um dos principais afluentes da 


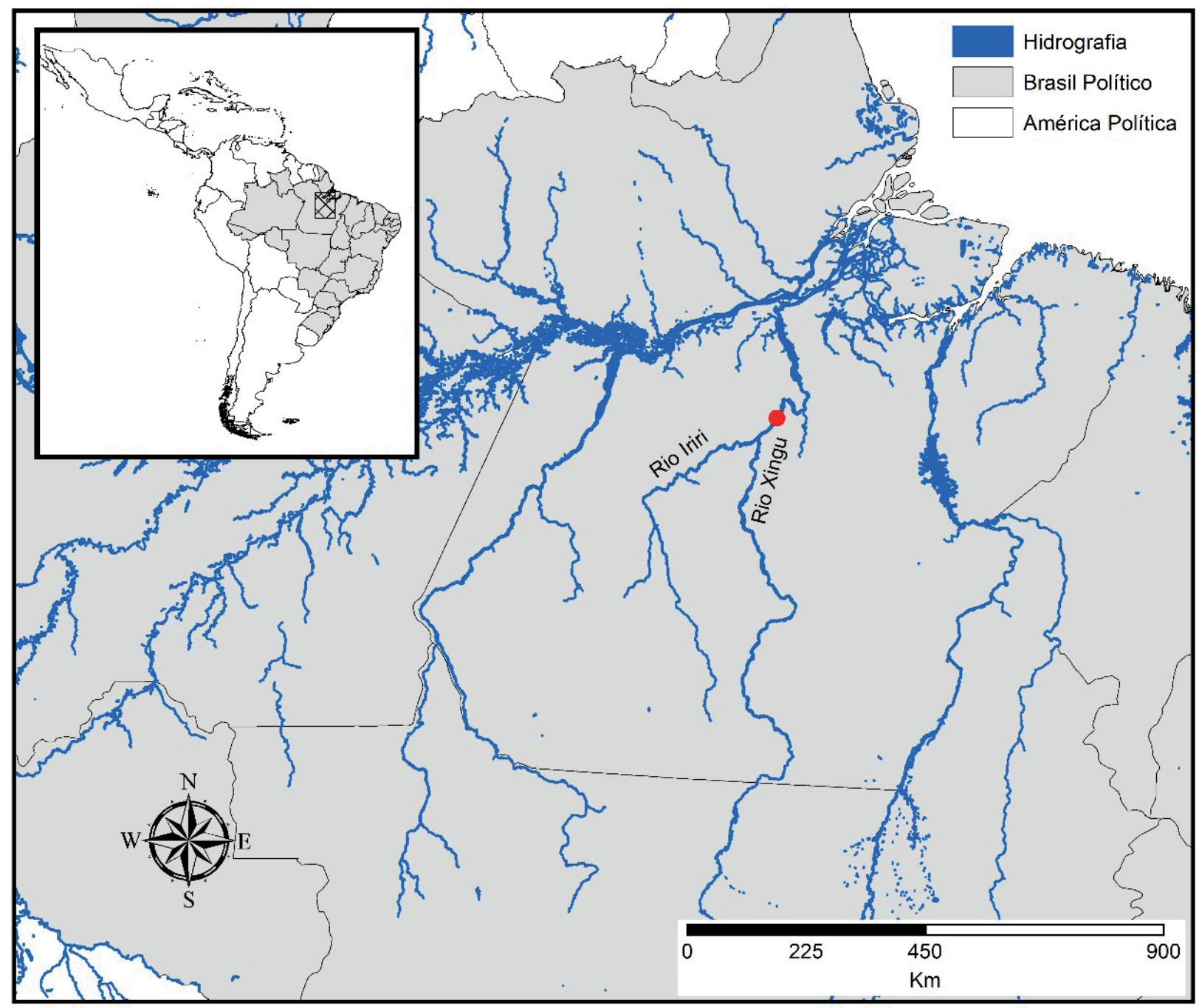

Figura 1. Localização da área de estudo em uma ilha no rio Xingu (círculo vermelho), a jusante da barragem de Pimental, no município de Vitória do Xingu, Pará.

Figure 1. Location of the study area on an island in the Xingu River (red circle), downstream of the Pimental dam, in the municipality of Vitória do Xingu, Pará.

margem direita do rio Amazonas, apresentando águas claras (Sioli 1984). O clima predominante da região, de acordo com a classificação de Köppen é do tipo "Amw" - Tropical quente e úmido (Peel et al. 2007), caracterizado por um período chuvoso, entre os meses de dezembro e maio, e um período seco, nos demais meses do ano. Essa ilha é predominantemente rochosa, fica submersa no período das chuvas e emerge no período da seca, isso faz com que as depressões contidas na parte rochosa da ilha retenham a água no período de seca. Portanto, por ser uma ilha pequena e que fica submersa tem as mesmas possibilidades de colonização por insetos aquáticos no interior e no litoral da ilha.

\section{Coleta de dados}

As coletas ocorreram em agosto de 2017, no período de seca, onde inicialmente, foi realizada uma análise visual em toda área da ilha para verificar os microhabitats disponíveis para comunidades de insetos aquáticos em fase imatura. Desta forma, foi identificada a predominância de dois microhabitats, um no interior da ilha, composto por poças com rocha como substrato predominante, e outro na parte litorânea, onde o substrato predominante era seixo (Figura 2). Com o auxílio de uma rede entomológica do tipo rapiché $(18 \mathrm{~cm}$ de diâmetro e rede de $250 \mu \mathrm{m}$ ) foram coletadas 60 amostras, sendo $30 \mathrm{em}$ poças no interior da ilha e 30 em seixos na zona litorânea da ilha. Para cada amostra a rede entomológica 


\section{a) Ilha}

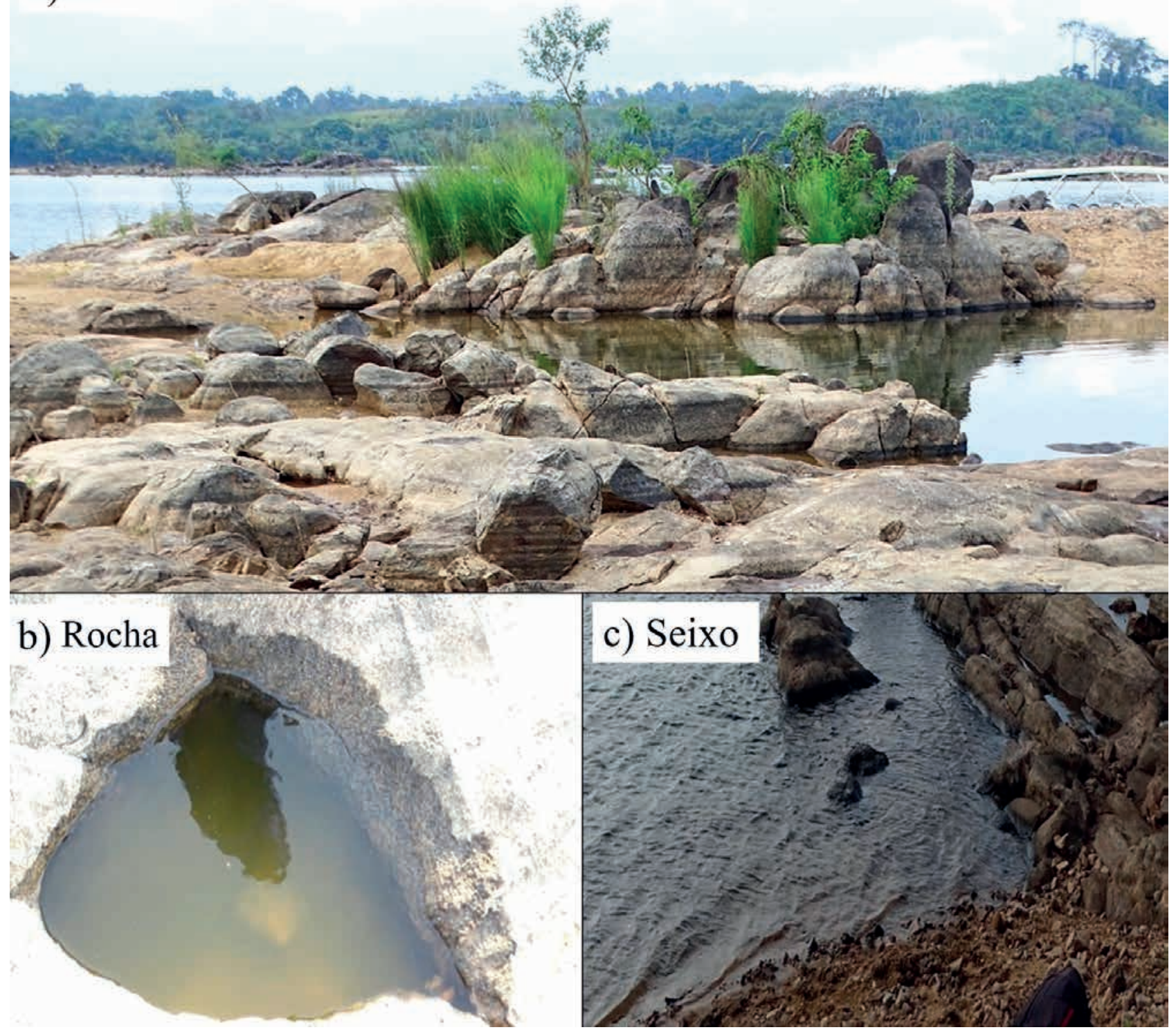

Figura 2. Foto panorâmica da ilha (a) com destaque para uma das poças onde foram coletadas as amostras interior com substrato de rocha (b) e para a região litorânea onde foram coletadas as amostras de seixo (c).

Figure 2. Panoramic photo of the island (a) with emphasis on one of the puddles where the interior samples with rock substrate were collected (b) and the coastal region where the pebble samples were collected (c).

foi passada três vezes e os substratos foram colocadas em bandejas brancas e os insetos foram triados por duas pessoas por 10 minutos, período necessário para que todos os insetos aquáticos das amostras fossem separados dos substratos. Posteriormente, os indivíduos foram fixados em solução de etanol $85 \%$, identificados ao nível de gênero com as chaves de Domínguez et al. (2006) e Pes et al. (2014). Consultas com especialistas e comparações com material depositado na Coleção Zoológica da Universidade Federal do Pará também foram realizadas. Para compreender a estrutura trófica das comunidades de insetos da ilha, os organismos foram classificados em grupos funcionais alimentares (GFA) baseado nos conhecimentos prévios disponíveis na literatura (Baptista et al. 2006, Dedieu et al. 2015, Castro et al. 2017, Brito et al. 2018).

\section{Análise de dados}

A riqueza de gêneros foi estimada por amostras utilizando Jackniffe de $1^{\text {a }}$ ordem (Heltshe \& Forrester 2009). A partir deste método, pretendeuse estimar a riqueza mais próxima do real, uma vez que é esperado que os métodos de coleta não 
consigam detectar todos os táxons, em especial os táxons mais raros. Em seguida, a riqueza estimada dos gêneros foi comparada entre os dois microhabitats distintos (litorâneos - lóticos, e de interior da ilha - lênticos) pela comparação de intervalos de confiança. Para testar as diferenças na abundância de indivíduos de ET entre os diferentes microhabitats foi realizado um teste $t$ com variâncias separadas (Zar 2010), uma vez que o pressuposto de homogeneidade de variância não foi atendido. Para testar a diferença na composição de gêneros entre as amostras diferentes microhabitats foi realizada uma Análise multivariada permutacional de variância usando matrizes de distância (PERMANOVA, Anderson 2005), e para verificar a variação da composição de gêneros entre os microhabitats, foi realizada uma Análise de homogeneidade multivariada de dispersões de grupos (PERMDISP, Anderson 2005). Ambas análises foram baseadas na matriz de dissimilaridade calculada pelo método de Bray-Curtis. Para visualizar graficamente o que foi testado na PERMANOVA e PERMDISP, utilizamos a mesma matriz de dissimilaridade, e ordenamos as comunidades em um espaço multivariado com uma Análise de Coordenadas Principais (PCoA). Utilizamos as funções t.test do pacote
Stats (Oksanen et al. 2019), e as funções adonis, betadisper e vegdist do pacote vegan (Oksanen et al. 2019), executadas no ambiente R (R Core Team 2019 -Version 3.0.2).

\section{RESULTADOS}

Foram coletados 56 indivíduos distribuídos em seis famílias (Coryphoridae, Leptohyphidae, Leptophlebiidae, Polymirtacyidae, Ecnomidae e Odontoceridae). Destes, 13 gêneros foram da ordem Ephemeroptera e dois gêneros de Trichoptera (ET). Nenhum Plecoptera foi amostrado. Os gêneros mais comuns foram Simothraulopsis Demoulin, 1966 e Campsurus Eaton, 1868 com 22 e 10 indivíduos, respectivamente (Tabela 1).

Baseado na inferência por intervalo de confiança, houve diferença entre a riqueza de gêneros estimada para rocha $(15,68 \pm 3,88)$ e seixo (11,64 $\pm 3,73$ ) (Figura $3 A)$. Em média, foram estimados quatro gêneros a mais para o microhabitat do interior da ilha (lêntico poças), com maior abundância nesse tipo de ambiente $\left(\mathrm{t}_{\text {variancia separada }}=4,948 ; \mathrm{gl}=42,452, \mathrm{p}<\right.$ 0,001). Em média, ocorreu um indivíduo a mais no microhabitat localizado no interior da ilha (lênticos) do que o encontrado no microhabitat
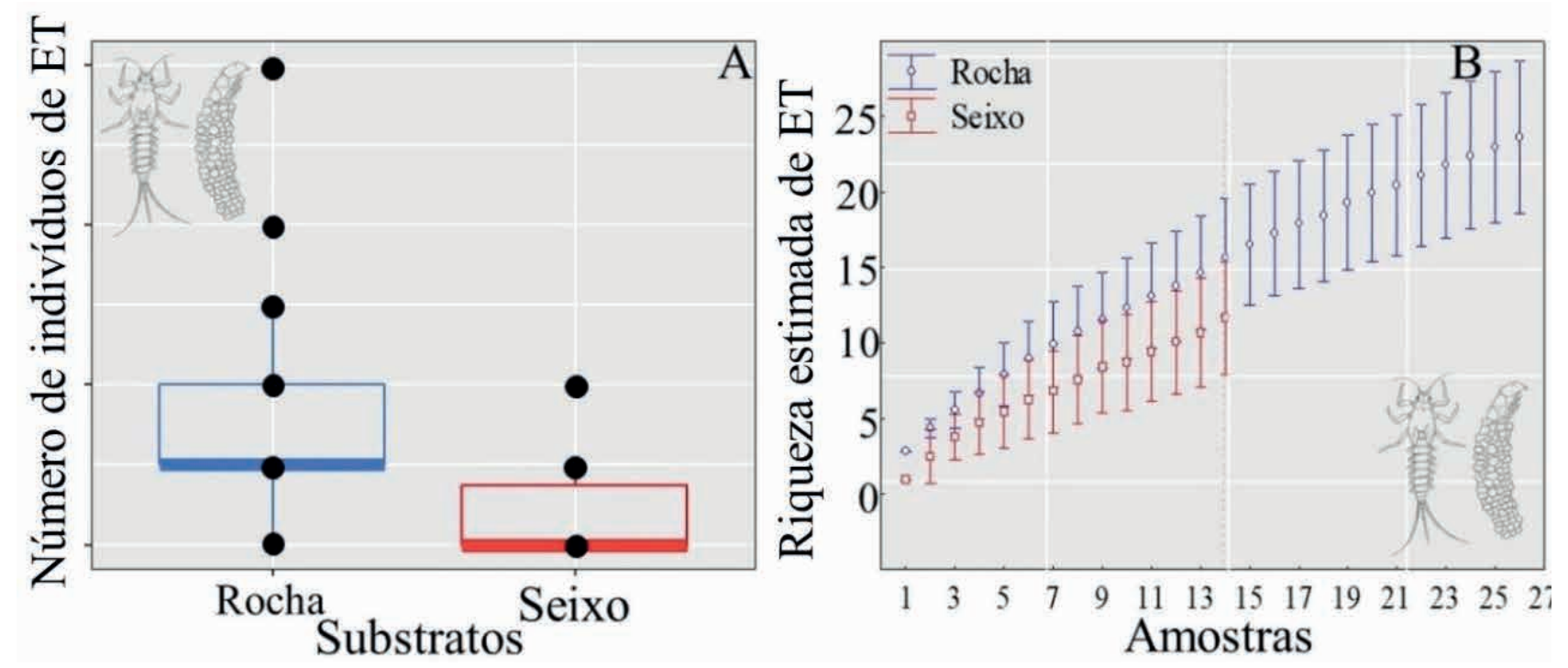

Figura 3. Boxplot com a comparação do número de gêneros estimados de imaturos de Ephemeroptera e Thichoptera (ET) entre microhabitats litorâneos (substrato seixo) e de interior da ilha (substrato rocha) (A), e número de indivíduos (mediana e desvio padrão) (B) de uma ilha do Rio Xingu, na Amazônia brasileira.

Figure 3. Boxplot with the comparison of the number of estimated genera of immature Ephemeroptera and Thichoptera (ET) between coastal microhabitats (pebble substrate) and interior of the island (rock substrate) (A), and number of individuals (median and standard deviation) (B) from an island on the Xingu River, in the Brazilian Amazon. 


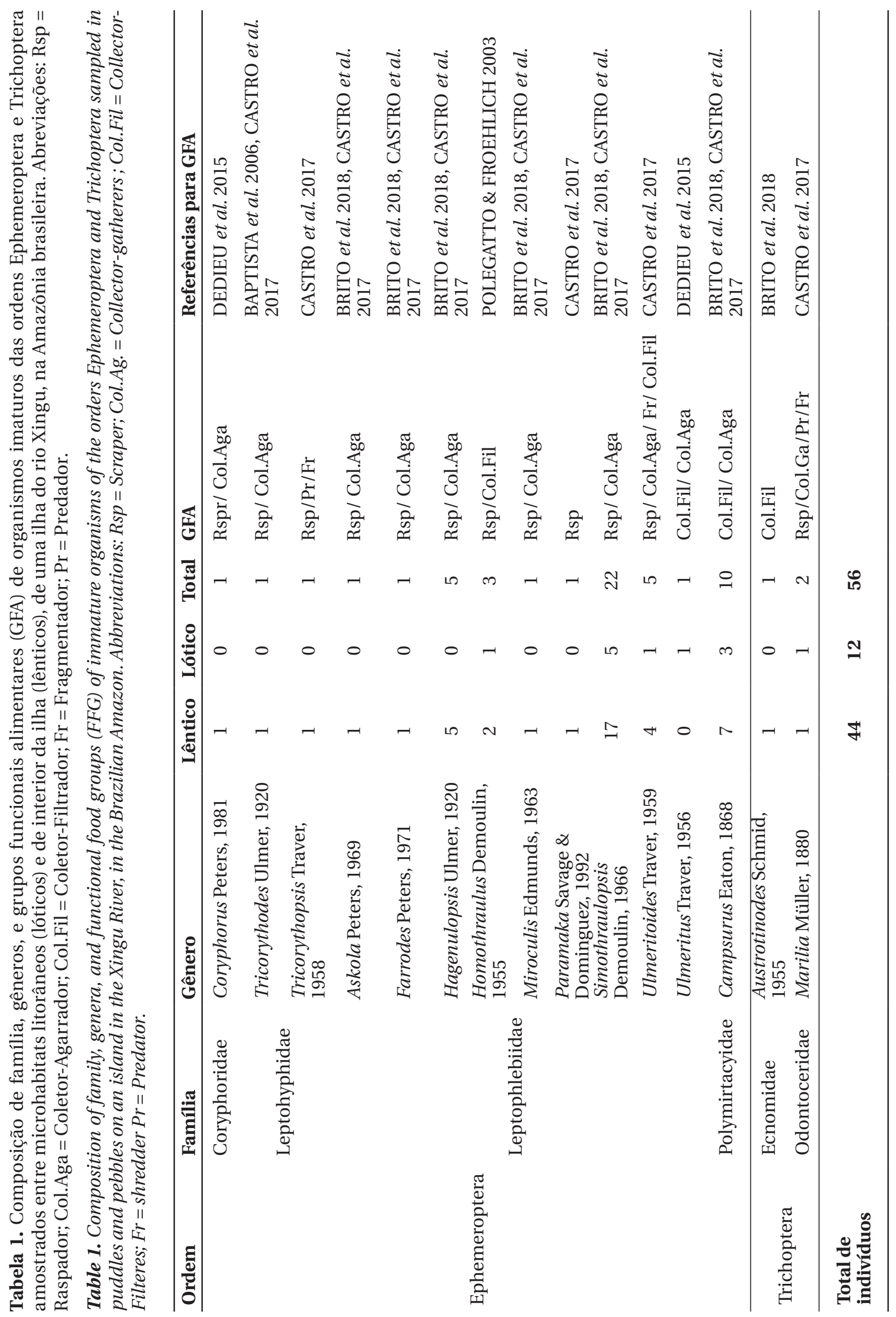


na região litorânea (lótico) (Figura 3B). Quando verificada a composição de gêneros de imaturos de ET, observamos que houve diferença tanto na composição (PERMANOVA; pseudo-F = 4,846; $\left.\mathrm{R}^{2}=0,077 ; \mathrm{p}<0,001\right)$, quanto na variação da composição (PERMDISP; $\mathrm{F}=46,219 ; \mathrm{p}<0,001$ ) entre os microhabitats de interior da ilha $(4,175) \mathrm{e}$ área litorânea $(0,842)$, sendo que a variabilidade de ET foi maior entre as poças do que as amostras da região litorânea com seixos.

Considerando todos os gêneros coletados encontramos apenas organismos raspadores, coletores-filtradores e coletores-agarradores (Tabela 1). No entanto, entre os nove gêneros coletados nas poças, todos eram raspadores e/ou coletores agarradores; enquanto o único gênero exclusivo da região litorânea com seixos era filtrador (Tabela 1).

\section{DISCUSSÃO}

Nosso estudo demonstrou que existiram diferenças nas comunidades de Ephemeroptera e Trichoptera entre os microhabitats em todos os aspectos investigados, como abundância, riqueza e composição de gêneros. O microhabitat litorâneo foi mais homogêneo na composição de gêneros. Nossas três predições foram corroboradas, em que o microhabitat no interior da ilha com substrato rocha apresenta maior riqueza de gêneros e abundância de indivíduos em comparação ao microhabitat na região litorânea da ilha com substrato seixo; e que a composição de gêneros difere entre os microhabitats, em virtude da distinta disponibilidade de recursos alimentares entre os microhabitats.

É possível que essa relação entre riqueza de gêneros e abundância de indivíduos exista devido ao microhabitat com substrato de rocha (lênticas) apresentar um ambiente com maior estabilidade que a região litorânea da ilha (lóticas), com substratos de seixo expostos a correnteza do rio, como encontrado em Duan et al. (2008) . Essas diferenças entre os microhabitats também podem ocorrer em virtude da distinta disponibilidade de recursos alimentares entre os microhabitats (Schmitt et al. 2020).

O microhabitat litorâneo apresentou predomínio de seixos como substrato, que se deslocam mais facilmente com a velocidade da água, não permitindo a fixação e permanência de organismos não adaptados a essas condições (Towsend \& Hildrew 1994), atuando como um filtro de espécies que pode tornar esse microhabitat mais homogêneo. Já nos ambientes lênticos do microhabitat no interior da ilha, a presença de substrato de rochoso pode favorecer maior heterogeneidade ambiental, sendo que as condições lênticas oferecem maior estabilidade ao ambiente (Giller \& Malmqvist 1998). As diferenças observadas na composição de gêneros indicaram que o ambiente pode determinar quais espécies ocorrem em cada local:

Organismos raspadores e coletores agarradores foram predominantes em ambos os microhabitats-. Segundo a Teoria do Rio Contínuo de Vannote et al. (1980), prediz que existe um gradiente de microhabitats da cabeceira até a foz do rio, desse modo, os grupos funcionais de macroinvertebrados podem se organizar de acordo com a ordem dos riachos. Em riachos de menor ordem, principalmente nas nascentes, há maior quantidade de material alócne proveniente da vegetação, desta forma, predomínio de macroinvertebrados fragmentadores (Vannote et al. 1980). Segundo esta mesma teoria, é esperado que em rios com maior ordem existam mais organismos coletores. No entanto, a classificação utilizada por Vannote não separa os coletores numa descrição mais fina, como coletores filtradores e coletores apanhadores. Utilizando uma classificação trófica um pouco mais refinada, Shimano et al. (2012) verificaram que a abundância de Ephemeroptera, de coletores filtradores e coletores apanhadores aumenta a partir dos riachos de $4^{\text {a }}$ ordem. Embora os riachos de $4^{\mathrm{a}}$ ordem estudados por Shimano et al. (2012) sejam rios menores que o Rio Xingu, nossos resultados corroboram essa evidência e indicam que os coletores são mais abundantes em rios a partir de $4^{\text {a }}$ ordem. Esse predomínio ocorre nos rios de ordem mais elevada, partículas de diferentes tamanhos são carregadas das margens e das porções a jusante, onde as maiores servem de alimento para coletores apanhadores, enquanto as menores são a base alimentar para coletores filtradores (Cummins 1974, Vannote et al. 1980, Cummins et al. 2005, Shimano et al. 2012, Brasil et al. 2014.

Entre os nove gêneros coletados 
exclusivamente nos ambientes lênticos (poças), apenas Austrotinodes pertence à ordem Trichoptera, os demais à Ephemeroptera e tiveram o hábito alimentar classificado como raspador. A predominância de raspadores nessas poças onde os substratos predominantes eram as rochas é relatada como uma relação direta com a disponibilidade de recurso alimentar para esses grupos, já que os raspadores se alimentam de perifíton aderido ao substrato, sobretudo a superfícies duras como rochas (Lock et al. 1984, Cummins et al. 2005). Por isso, em microhabitats como poças lênticas expostas a grande quantidade de luz é esperado um aumento de produção autóctone, o que disponibilizada grandes quantidades de alimento para os raspadores aderido as rochas (Vannote et al. 1980, Hershey et al. 2010, Brasil et al. 2014). Por isso, esses organismos desempenham um papel importante consumindo e controlando a biomassa de organismos produtores (Feminella \& Hawkins 1995, Biggs et al. 1998).

Mesmo se tratando de ambientes diferentes, vale destacar que as comunidades de insetos aquáticos do Rio Xingu aparentemente têm uma densidade de indivíduos e número de grupos funcionais alimentares menor do que é encontrado em outros estudos que investigaram riachos amazônicos, que reportaram em média 40 gêneros e cinco grupos funcionais alimentares - GFA (i.e. Faria et al. 2017, Luiza-Andrade et al. 2017). Grandes rios costumam possuir reduzida heterogeneidade de substratos orgânicos, o que faz com que os poucos insetos que persistem nesses ambientes, necessitem ser adaptados a áreas abertas com grande vazão e maior disponibilidade de habitat inorgânico do que orgânico (Statzner \& Higler 1986, Townsend 1989). A estrutura física é um componente importante na estruturação dos insetos aquáticos, e o ambiente atua como um agente de seleção natural limitando a persistência de espécies que possuem requerimentos de hábitats específicos (Southwood 1977). No entanto, vale a ressalva que nossa amostragem é eficiente apenas para comparação entre microhabitats, não sendo representativo para compreender aspectos gerais da diversidade do rio Xingu.

Este foi um estudo de carácter descritivo que indicou que a heterogeneidade ambiental gerada pelos diferentes microhabitats pode produzir condições e recursos e dessa forma ser determinante para maior diversidade de insetos aquáticos. Mais estudos sobre esta diferença são necessários, uma vez que manter essa diversidade de substratos pode ser importante para a estrutura trófica do rio como um todo, uma vez que-afetaria a diversidade de insetos aquáticos e esses organismos são base da cadeia trófica local.

\section{AGRADECIMENTOS}

Todos os autores agradecem ao PROAP-Capes (processo 001) destinado ao PPGBC-UFPA em 2017 pelo financiamento das coletas de campo. ALA agradece ao CNPq pela concessão de bolsa de doutorado (141991/2016-0) e SRS agradece a Capes pela concessão de bolsa de mestrado. LSB agradece a Capes pela concessão de bolsa de Pós-Doutorado PNPD vinculado ao PPGZOOL da UFPA.

\section{REFERÊNCIAS}

Allan, J. D. 1995. Stream ecology. Structure and function of running waters. Chapman \& Hall, London. p. 388.

Anderson, M. 2005. PERMANOVA, Permutational multivariate analysis of variance. A computer program. Wiley StatsRef: Statistics Reference Online. DOI: 10.1002/9781118445112.stat07841

Anderson, M. J. 2005. PERMANOVA: a FORTRAN computer program for permutational multivariate analysis of variance. Departmen of Stattisitics. Department of Statistics.

Angeli, K. B., Rozário, E. M. M., \& Salles, F. F. 2015. Checklist of Ephemeroptera (Insecta) from São Mateus River Basin, Espírito Santo, Brazil. Revista Brasileira de Entomologia, 59(3), 197204. DOI: 10.1016/j.rbe.2015.06.004

Baptista, D. F., Buss, D. F., Dias, L. G., Nessimian, J. L., Da Silva, E. R., De Moraes Neto, A. H. A., De Carvalho, S. N., De Oliveira, M.A., \& Andrade, L. R. 2006. Functional feeding groups of Brazilian Ephemeroptera nymphs: Ultrastructure of mouthparts. Annales de Limnologie, 42(6), 8796. DOI: $10.1051 / \mathrm{limn} / 2006013$

Baptista, D. F., Dorvillé, L. F., Buss, D. F., \& Nessiamian, J. L. 2001. Spatial and temporal organization of aquatic insects' assemblages in the longitudinal gradient of a tropical river. 
Revista Brasleira de Biologia, 61(2), 295-304. DOI: 10.1590/s0034-71082001000200012

Barlow, J., França, F., Gardner, T. A., Hicks, C. C., Lennox, G. D., Berenguer, E., Castello, L., Economo, E.P., Ferreira, J., Guénard, B., Gontijo Leal, C., Isaac, V., Lees, A. C., Parr, C. L., Wilson, S. K., Young, P. J., \& Graham, N. A. J. 2018. The future of hyperdiverse tropical ecosystems. Nature, 559, 517-526. DOI: 10.1038/s41586-0180301-1

Biggs, B. J. F., Stevenson, R. J., \& Lowe, R. L. 1998. A habitat matrix conceptual model for stream periphyton. Archiv Fur Hydrobiologie, 143(1), 21-56. DOI: 10.1127/archivhydrobiol/143/1998/21

Brasil, L. S., Juen, L. Batista, J. D., Pavan, M. G. \& Cabette, H. S. R. 2014. Longitudinal Distribution of the Functional Feeding Groups of Aquatic Insects in Streams of the Brazilian Cerrado Savanna. Neotropical Entomology, 43(5), 421-428. DOI: 10.1007/s13744-014-02349.

Brito, J. G., Martins, R. T., Oliveira, V. C., Hamada, N., Nessimian, J. L., Hughes, R. M., Ferraz, S. F. B., \& de Paula, F. R. 2018. Biological indicators of diversity in tropical streams: Congruence in the similarity of invertebrate assemblages. Ecological Indicators, 85, 85-92. DOI: 10.1016/j. ecolind.2017.09.001

Castro, D. M. P., Dolédec, S., \& Callisto, M. 2017. Landscape variables influence taxonomic and trait composition of insect assemblages in Neotropical savanna streams. Freshwater Biology, 62(8), 1472-1486. DOI: 10.1111/ fwb.12961

Cummins, KW, \& Klug, M. J. 1979. Feeding Ecology of Stream Invertebrates. Annual Review of Ecology and Systematics, 10(1),147-172. DOI: 10.1146/annurev.es.10.110179.001051

Cummins, K W., \& Lauff, G. H. 1969. The influence of substrate particle size on the microdistribution of stream macrobenthos. Hydrobiologia, 34(2), 145-181. DOI: 10.1007/ BF00141925

Cummins, K W., Merritt, R. W., \& Andrade, P. C. N. 2005. The use of invertebrate functional groups to characterize ecosystem attributes in selected streams and rivers in south Brazil. Studies on Neotropical Fauna and Environment, 40(1), 69-89. DOI: 10.1080/01650520400025720
Cummins, K. W. 1974. Structure and function of stream ecosystems. BioScience, 24(11), 631641.

De Marco, P., \& Vianna, D. M. 2005. Distribuição do esforço de coleta de Odonata no Brasil Subsídios para escolha de áreas prioritárias para levantamentos faunísticos. Lundiana, 6 , 13-26.

Dedieu, N., Rhone, M., Vigouroux, R., \& Céréghino, R. 2015. Assessing the impact of gold mining in headwater streams of Eastern Amazonia using Ephemeroptera assemblages and biological traits. Ecological Indicators, 52, 332-340. DOI: 10.1016/j.ecolind.2014.12.012

Domínguez, E. 2006. Ephemeroptera de América Del Sur. 2nd ed. Bulgaria: Pensoft Publishers: p. 646.

Duan, X., Wang, Z. \& Tian, S. 2008. Effect of streambed substrate on macroinvertebrate biodiversity. Frontiers of Environmental Science \& Engineering in China, 2(1), 122-128. DOI: $10.1007 / \mathrm{s} 11783-008-0023-y$

Faria, A. P. J., Ligeiro, R., Callisto, M., \& Juen, L. 2017. Response of aquatic insect assemblages to the activities of traditional populations in eastern Amazonia. Hydrobiologia, 802, 39-51. DOI: 10.1007/s10750-017-3238-8

Feminella, J. W., \& Hawkins, C. P. 1995. Interactions between stream herbivores and periphyton: A quantitative analysis of past experiments. Journal of the North American Benthological Society, 14(4), 465-509. DOI: 10.2307/1467536

Giller, P.S. \& Malmqvist, B. 1998. The Biology of streams and rivers: Biology of Habitat. Oxford: Oxford University Press: p. 96.

Heltshe, J. F., \& Forrester, N. E. 1983. Estimating species richness using the jackknife procedure. Biometrics, 39(1), 1-11. DOI: 10.2307/2530802

Hershey, A. E., Lamberti, G. A., Chaloner, D. T., \& Northington, R. M. 2010. Aquatic insect ecology. In Ecology and classification of North American freshwater invertebrates. Academic Press: p. 659.

Hickisch, R., Hodgetts, T., Johnson, P. J., SilleroZubiri, C., Tockner, K., \& Macdonald, D. W. 2019. Effects of publication bias on conservation planning. Conservation Biology, 33, 1151-1163. DOI: 10.1111/cobi.13326

Juen, L., Brasil, L. S., Salles, F. F., Batista, J. D., \& Cabette, H. S. R. 2017. Mayfly assemblage 
structure of the Pantanal Mortes-Araguaia flood plain. Marine and Freshwater Research, 68(11), 2156-2162. DOI: 10.1071/ MF17013

Lees, A. C., Peres, C. A., Fearnside, P. M., Schneider, M., \& Zuanon, J. A. S. 2016. Hydropower and the future of Amazonian biodiversity. Biodiversity and Conservation, 25, 451-466. DOI: $10.1007 /$ s10531-016-1072-3

Lock, M., Costerton, J., Ventullo, R., Wallace, R., \& Charlton, S. 1984. River epilithon: toward a structural-functional model. Oikos, 42(1), 1022.

Luiza-Andrade, A., Brasil, L. S., Benone, N. L., Shimano, Y., Farias, A. P. J., Montag, L. F., Dolédec, S., \& Juen, L. 2017. Influence of oil palm monoculture on the taxonomic and functional composition of aquatic insect communities in eastern Brazilian Amazonia. Ecological Indicators, 82, 478-483. DOI: 10.1016/j.ecolind.2017.07.006

Magalhães, S. B. \& Hernándes, F. M. 2009. Painel de Especialistas: análise crítica do Estudo de Impacto Ambiental do Aproveitamento Hidrelétrico de Belo Monte. Belém: p. 230.

Merritt, R. W. \& Cummins, K.W. 1996. An introduction to the aquatic insects of north America. 2nd ed. Dubuque: Kendall Hunt Publishing Co: p. 826.

Miguel, T. B., Calvão, L. B., Vital, M. V. C., \& Juen, L. 2017. A scientometric study of the order Odonata with special attention to Brazil. International Journal of Odonatology. 20(1), 1-16. DOI: 10.1080/13887890.2017.1286267

Minshall, G.W. \& Minshall, J.N. 1977. Microdistribution of benthic invertebrates in a Rocky Mountain (U.S.A.) stream. Hydrobiologia 55, 231-249.

Norte Energia AS. 2011. PBA: Plano básico ambiental, versão final, Setembro de 2011. Norte Energia SA,: Rio de Janeiro, Brazil: 7 vols. + annexes.

Oksanen, J., Guillaume Blanchet, F., Friendly, M., Kindt, R., Legendre, P. \& McGlinn, D. 2019. vegan: Community Ecology Package. R package version 2.5-6. Vienna: R Foundation for Statistical Computing. https://CRAN.Rproject.org/package=vegan

Peel, M. C., Finlayson, B. L., \& McMahon, T. A. 2007. Updated world map of the Köppen-Geiger climate classification. Hydrology and Earth System Sciences. DOI: 10.5194/hess-11-16332007

Pes, A.M., Moreira Santos, A.P., Barcelos-Silva, P. \& Camargos, L.M. 2014. Ordem Trichoptera. In: N. Hamada JL, Nessimian J \& Querino RB (Eds.), Insetos Aquáticos na Amazônia Brasileira: taxonomia, biologia e ecologia. pp. 391-433. Manaus: Editora do INPA: p. 724

R Core Team. 2019. R: A language and environment for statistical computing (Version 3.0. 2) [Computer software]. $\mathrm{R}$ Foundation for Statistical Computing, Vienna, Austria. URL https://www.R-project.org/.

Rosenberg, V. H., Resh, D. M. 1993. Freshwater biomonitoringandbenthicmacroinvertebrates. 1st ed. New York: Chapman \& Hall: p. 488.

Resh, V. H., \& Unzicker, J. D. 1975. Water quality monitoring and aquatic organisms: the importance of species identification. Journal of the Water Pollution Control Federation. 47(1), 9-19.

Schmitt, R., Silva, A. L. L., Soares, L. C. M., Petrucio, M. M. \& Siegloch, A. E. 2020. Influence of microhabitat on diversity and distribution of Ephemeroptera, Plecoptera, and Trichoptera in subtropical forest streams. Studies on Neotropical Fauna and Environment, 55(2), 129138. DOI: $10.1080 / 01650521.2019 .1704984$

Shimano, Y., Salles, F. F., \& Juen, L. 2013. Study of the mayfly order Ephemeroptera (insecta) in Brazil: A scienciometric review. Revista Brasileira de Entomologia, 57, 359-364. DOI: 10.1590/S0085-56262013005000043

Shimano, Y., Salles, F. F., Faria, L. R. R., Cabette, H. S. R., \& Nogueira, D. S. 2012. Distribuição espacial das guildas tróficas e estruturação da comunidade de Ephemeroptera (Insecta) em córregos do Cerrado de Mato Grosso, Brasil. Iheringia - Serie Zoologia, 102(2), 187-196. DOI: 10.1590/S0073-47212012000200011

Sioli, H. 1984. The Amazon: limnology and landscape ecology of a mighty tropical river and its basin. Internationale Revue der Gesamten Hydrobiologie und Hydrographie, 56nd ed: p. 762.

Southwood, T. R. E. 1977. Habitat as the template for ecological strategies? The Journal of Animal Ecology, 46, 336-365. DOI: 10.2307/3817 
Statzner, B., \& Higler, B. 1986. Stream hydraulics as a major determinant of benthic invertebrate zonation patterns. Freshwater Biology, 16(1), 127-139. DOI: $\quad 10.1111 / j .1365-2427.1986$. tb00954.x

Strahler, A. N. 1957. Quantitative analysis of watershed geomorphology. Transactions of the American geophysical Union, 38(6), 913-920. DOI: 10.1029/TR038i006p00913

Thorp J.H., Thoms M.C. \& Delong M.D. 2006. The riverine ecosystem synthesis: biocomplexity in river networks across space and time. River Research and Applications, 147, 123-147.

Townsend, C. 1989. The patch dynamics concept of stream community ecology. Journal of the North American Benthological Society, 8(1), 36-50. DOI:10.2307/1467400

Townsend, C. R., \& Hildrew, A. G. 1994. Species traits in relation to a habitat templet for river systems. Freshwater Biology, 31(3), 265-275. DOI: $10.1111 /$ j.1365-2427.1994.tb01740.x

Vannote, R. L., Minshall, G. W., Cummins, K. W., Sedell, J. R., \& Cushing, C. E. 1980. The river continuum concept. Canadian Journal of Fisheries and Aquatic Sciences, 37(1), 130-137. DOI: 10.1139/f80-017

Wallace, J.B., \& Anderson, N. H. 1995. Habitat, life history, and behavioral adaptations of aquatic insects, In: Merritt, R.W., and K.W. Cummins (eds.), An Introduction to the Aquatic Insects of North America: pp. 41-73. Dubuque, Iowa: Kendall/Hunt Publishing Company.

Whittaker, R. J., Araújo, M. B., Jepson, P., Ladle, R. J., Watson, J, E. M., \& Willis, K. J. 2005. Conservation Biogeography: Assessment and Prospect. Diversity and Distributions, 11(1), 3-23. DOI: 10.1111/j.1366-9516.2005.00143.x

Zar, J. H. 2010. Biostatistical Analysis. 5nd ed. Upsaddler Road: Prentice Hall: p. 944.

Submitted: 03 April 2020 Accepted: 17 November 2020 Published on line: 04 December 2020 Associate Editor: Barbara Dunck 